\title{
From Data Silos to Intelligent Web for RSO Recognition
}

\author{
Bin Liu ${ }^{\mathrm{a}}$, Li Yao ${ }^{\mathrm{a},}$, Junfeng $\mathrm{Wu}^{\mathrm{a}}$, Zhiyong Hao ${ }^{\mathrm{b}}$, Zheyuan Ding ${ }^{\mathrm{a}}$ \\ ${ }^{a}$ College of System Engineering, National University of Defense Technology, Changsha 410073, China \\ ${ }^{\mathrm{b}}$ Shenzhen University, Shenzhen 518060, China \\ *Corresponding Author: 913381959@qq.com
}

\begin{abstract}
Due to the lack of data caused by limited and uncertain observations, how to classify Resident Space Object (RSO) remains to be a difficult problem. Previous RSO classifications mainly focus on the problem when the "hard data" which are obtained by physical sensors are missing. They make use of features extracted from observation data which center on RSOs themselves only and are still very limited. This paper proposes to use an RSO Ontology named OntoStar to represent hard data and soft data. This representation not only describes RSOs themselves, but also links related objects to RSOs, establishing a more comprehensive and accurate RSO description to support more accurate and robust classifications. OntoStar not only contains mined feature deducting rules to refine the RSO feature information, but also includes a variety of mined RSO recognition rules to classify RSOs based on different sets of features. Experimental results show that RSO classification based on OntoStar can effectively solve the RSO classification problem under limited or uncertain observation conditions.
\end{abstract}

Keywords: space objects, recognition, multi-source, fusion, soft data, ontology.

\section{Introduction}

Resident Space Object (RSO) recognition plays an important role in space exploration, protection of space assets, and especially space situational awareness ${ }^{(1-3)}$. The RSO classification task refers to the classification of space objects such as satellites, debris, and rockets in the earth's orbit based on observed data ${ }^{(2)}$. The classification here includes identifying the type, mission, and purpose of the RSO. However, due to limited or uncertain observations, accurate classification of RSO remains a problem ${ }^{(3-5)}$.
Probabilistic reasoning on a RSO taxonomy are conducted to classify RSOs from coarse to fine when RSO features are incomplete ${ }^{(5)}$. Ontology-based classification uses feature deducting rules to estimate missing features, and obtains various RSO classification rules through machine learning to enrich the RSO classification knowledge base ${ }^{(2)}$. With these rules, ontology-based RSO classification obtains better results than other methods under the condition that some features are missing. The above methods mainly focus on solving the problem of RSO classification when the physical "hard sensor" data is missing. They mainly use data about RSOs themselves which are still very limited.

In space situational awareness, integrating data from multiple sources is an important solution for extending RSO observations ${ }^{(6,7)}$. Sensor data can be divided into "hard sensor" data and "soft sensor" data, also known as hard data and soft data ${ }^{(1)}$. Hard data refer to the observation data obtained by sensors in real time. Soft data refer to the relevant information generated by humans ${ }^{(8,9)}$, including reports and open source information on the Internet ${ }^{(10)}$, which can provide contextual information related to the object, making object recognition no longer limited to the features of objects themselves. As a useful complement to hard data, soft data have gained increasing attention in recent years, because it can expand the source of sensing data and provide contextual information ${ }^{(8,10-13)}$. Soft and hard data fusion is a new topic of information fusion in recent years ${ }^{(14)}$. In fact, there is much soft sensing information about RSO that can be used for RSO recognition, such as the RSO anomaly status posted by operators on the Internet ${ }^{(1)}$, space collision incident reports, analysis of spacecraft launch bases, and reports on satellite launches.

Ontologies have many applications in integrating diverse data and representing multiple types of knowledge 
$(1,2,15,16)$, especially in the field of space situational awareness, with its unique advantages. For example, ontologies are used to represent soft and hard data for buildings, and cross-domain data islands are integrated to achieve optimal performance of building performance ${ }^{(12)}$. In space situational awareness domain, the space object ontology can integrate multi-source RSO data ${ }^{(15,16)}$, represent a variety of RSO features, and enhance RSO characterization and provide robust entity tracking ${ }^{(15)}$. OntoStar (Ontology of Space Target Automatic Recognition) integrates domain knowledge and knowledge obtained by machine learning such as tactics, regulations, and inductive rules estimating specific features, targeting at solving RSO classification problem when some features are missing ${ }^{(2)}$. At the same time, the ontologies of linked multi-source data are represented, which can be used in semantic rule mining methods to obtain hidden relational patterns, background knowledge, and interesting and useful features $^{(17)}$.

This paper proposes to use a unified ontology framework to represent RSO's multi-source data and knowledge. First, OntoStar represents RSO soft data and hard data to link RSO data silos, and expands the capabilities of RSO data representation to enable ontology-based object recognition systems to obtain more complete and accurate RSO descriptions. Then, rule learning methods obtain feature deducting rules, further enriching knowledge of OntoStar to derive more interesting and useful features of RSO. Meanwhile, multiple classification rules $^{(18)}$ for each RSO class are learned. These rules have the same conclusion but different preconditions, making it possible to classify an RSO based on different sets of features. On this basis, RSOs are classified based on reasoning on OntoStar in two phases. Firstly, OntoStar is used to represent the hard data of RSO and to reason RSO's type. Secondly, if hard data are not enough to identify RSO's type completely, soft data will be represented by OntoStar in reasoning RSO's type.

This paper has three main contributions. Firstly, the advantages of ontology in integrating multi-source data and multiple types of knowledge are fully made use of. Secondly, soft data and hard data are represented and integrated using OntoStar, obtaining a complete and more accurate RSO description. RSO feature deducting rules can be obtained from the integrated soft data and hard data, to derive more interesting and useful features of RSO. More diverse RSO classification rules can also be learned to enable RSO classification based on different sets of features, so that robust RSO classification can be done even if some features are missing. Thirdly, two-phase RSO classification is proposed to fully utilize the characteristics of hard data and soft data, aiming at classifying RSOs under circumstances of limited and uncertain observations.

\section{RSO Recognition Based on Data Web}

Using OntoStar to represent RSO soft data and hard data from multiple sources and linking RSO data silos have two main purposes: one is to obtain complete and more accurate RSO descriptions, the other is to learn semantic rules to enrich knowledge in OntoStar using the linked data. The data form a semantic web which characterizes RSOs themselves and the relationship between the RSOs and other entities. Using OntoStar as the knowledge base to reason on the RSO data web, more interesting and useful RSO features are obtained. When reasoning on OntoStar, there are many paths that can derive an RSO's type, making RSO classification not rely on only a specific set of features any more.

The semantic web can be constructed by obtaining RSO soft data from reliable sources, and representing soft data and hard data using OntoStar.

\subsection{Collecting Soft RSO Data from Multiple Sources}

In addition to obtaining sensing data from space surveillance network for RSOs, RSO data can be accessed from many organizations and individuals who publish RSO data. RSO data from reliable sources can be used to describe and classify RSOs. Table 1 summarizes the data sources that provide RSO soft data.

Table 1. Multi-source RSO soft data.

\begin{tabular}{|c|c|}
\hline source & Information useful in RSO recognition \\
\hline $\begin{array}{c}\text { NASA } \\
\text { Spacecraft }\end{array}$ & $\begin{array}{c}\text { user, purpose, dry mass, power, launch time, launch } \\
\text { site, launch vehicle, etc. }\end{array}$ \\
\hline $\begin{array}{c}\text { CEOS } \\
\text { Payload }\end{array}$ & $\begin{array}{c}\text { purpose, power, type, } R \& D \text { status, } R \& D \text { organization } \\
\text { and technical parameters, etc. }\end{array}$ \\
\hline $\begin{array}{c}\text { UCS } \\
\text { Satellite }\end{array}$ & $\begin{array}{l}\text { country, owner, user, purpose, orbit type, orbit class, } \\
\text { ascension right ascension, period, inclination, dry mass, } \\
\text { launch mass, power, launch time, launch site, launch } \\
\text { vehicle, life expectancy, contractor, etc. }\end{array}$ \\
\hline CelesTrack & $\begin{array}{c}\text { period, inclination, perigee, apogee, eccentricity, radar } \\
\text { cross-sectional area and payload }\end{array}$ \\
\hline CalSky & $\begin{array}{c}\text { radar cross-sectional area, shape, length, width, height } \\
\text { and radius, etc. }\end{array}$ \\
\hline
\end{tabular}

In addition to the space object data in Table 1 , RSO 
historical data can also be used as soft data for RSO recognition. Other public data of space object include: space object surveillance data and Two Line Elements (TLE) data from North American Aerospace Defense Command (NORAD), radar cross-sectional area of RSO from Air Force Space Command (AFSPC) or online satellite monitoring platform (n2yo).

\subsection{Constructing RSO Data Web}

Due to barriers made by humans or caused by technical limitations, the multi-source RSO data form "data silos"(15,16). The biggest advantage of RSO domain ontology is that the ontology represents multi-source data to find a way out of the "data silos" dilemma ${ }^{(15)}$. OntoStar owns this advantage too. It represents data to form a "semantic web" which links multi-source data and centers on the RSO.

When OntoStar is used to represent RSO multi-source data, RSO instance is represented by Individual, and the external features of RSO are treated as feature instances to be also represented by Individuals. Relations between these instances are described by Object Property. The internal features of RSO instances and feature instances are represented by Data Property. In this way, a linked web centering on the RSO is established, describing the relations (external properties) between the RSO and its related entities, and the intrinsic properties of the RSO itself. Fig 1 shows some relations between the RSO named 2006-018A and instances/concepts represented by OntoStar.

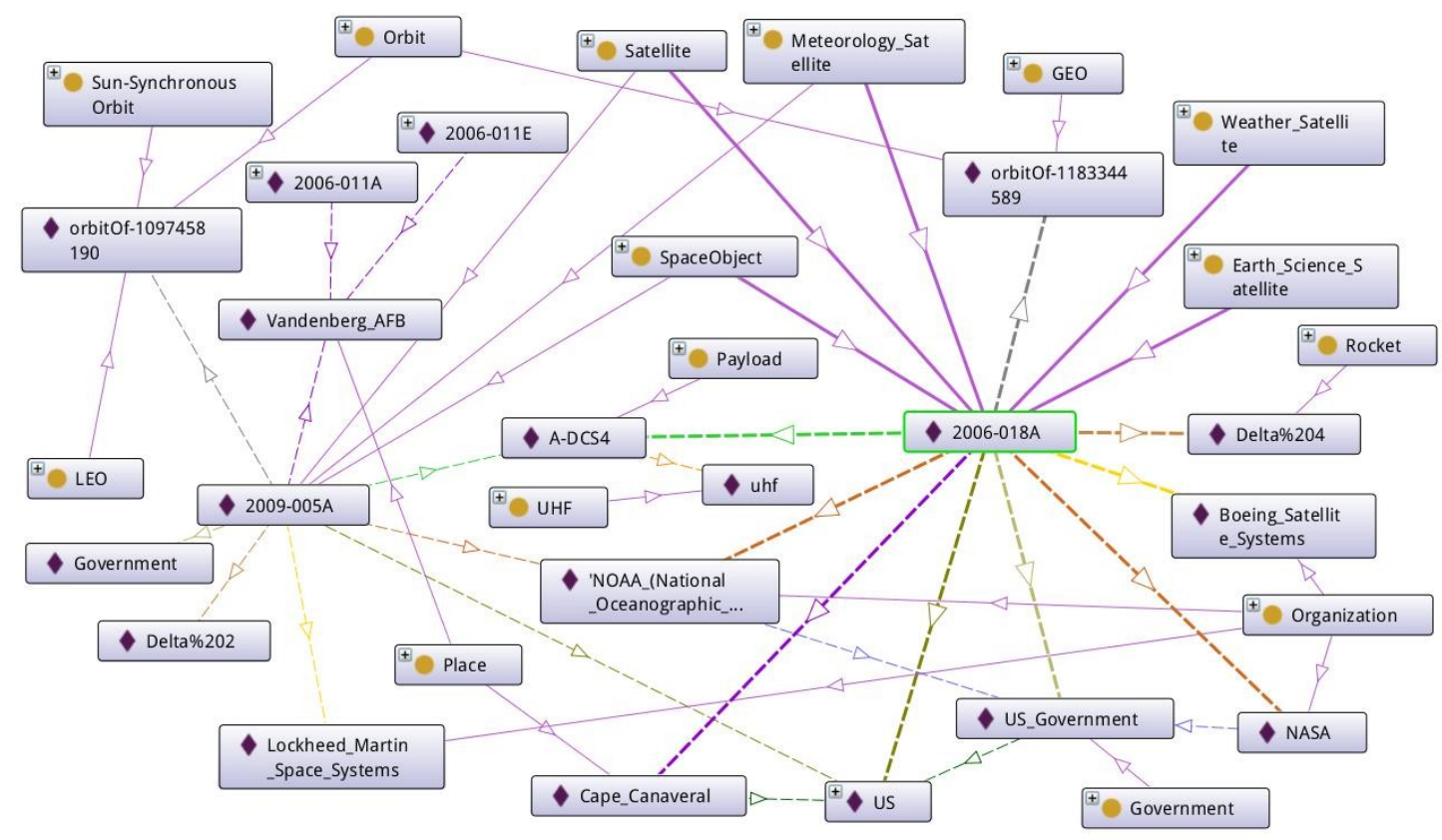

Fig. 1. Multi-source data of 2006-018A represented by OntoStar forms a "semantic web".

In OntoStar, data from different sources mainly describe different feature instances. E.g., data of CelesTrack and TLE describe orbit. Data from CEOS describe payloads. Data from NORAD Catalog, UCS Satellite and CalSky are mainly the features describing RSOs themselves. In Fig 1, it can be seen that 2006-018A's orbit is orbitOf-1183344589. Data Properties about the orbit are parameters from TLE. It can also be seen that orbitOf-1183344589 is a kind of GEO (Relation between instance and its type is described by $I s-A$ marked by arrow with solid line). A payload of 2006-018A is A-DCS4 described by data from CEOS. 2006-018A is linked with orbitOf-1183344589 and A-DCS4 using Object Properties "inOrbit" (marked by dark blue arrows with dotted lines) and "hasPayload" (marked by green arrow with dotted line), so that 2006-018A is the center for accessing data about objectOf-1183344589, A-DCS4 and 2006-018A. 2006-018A, orbitOf-1183344589 and A-DCS4 are described in Figures 2(a), (b), and (c), respectively.

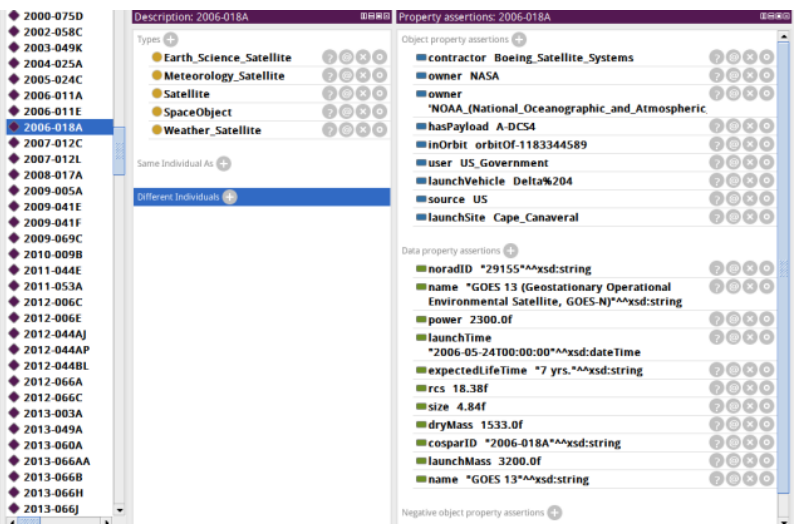

(a) representing 2006-018A using OntoStar 


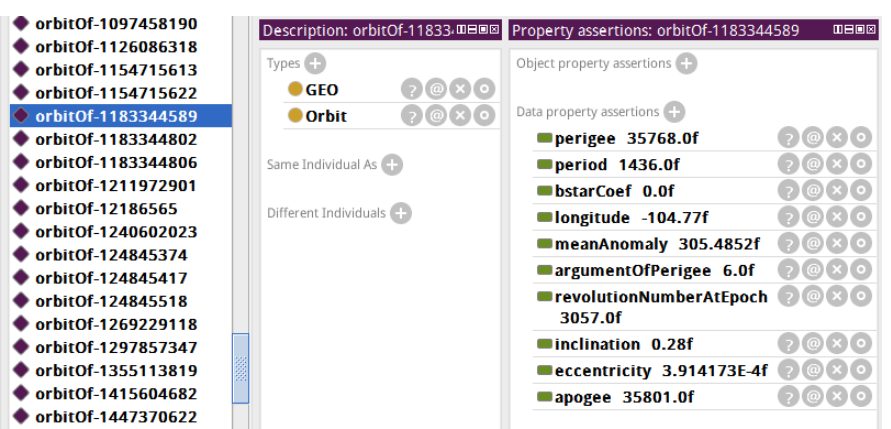

(b) representing orbitOf-1183344589 using OntoStar

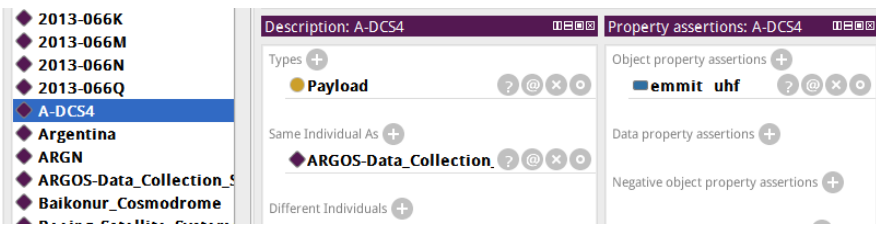

(c) representing A-DCS4 using OntoStar

Fig. 2. Describing instances using OntoStar.

As shown in Fig 2, internal features of 2006-018A are represented by Data Properties such as rcs, size, power, quality, and etc. Internal features of orbitOf-1183344589 are perigee, apogee, inclination, eccentricity, and etc. A-DCS4 emits uhf.

After using OntoStar to represent the RSO data, the ontology query tools can be used to access the RSO data. E.g., Protégé provides an OWL-DL statement to query the ontology. Fig 3 shows how to use DL to query the RSO who sources US.

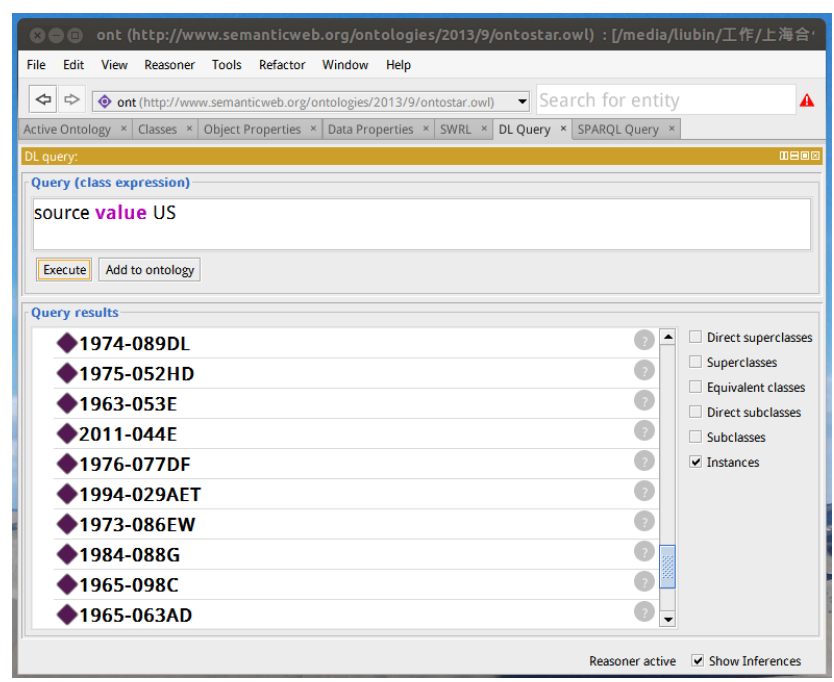

Fig. 3. Use DL to query RSO who sources from "US".

The DL query tool only supports class expressions. When complex relations are involved in the query, SPARQL will be needed ${ }^{(19)}$. E.g., to query RSOs who are launched at the same launch site which launches RSOs who own the same payload as 2006-018A does, the following links are necessary: 2006-018A $\rightarrow$ payload $\rightarrow$ RSO $\rightarrow$ launch site $\rightarrow$ RSO. Using SPARQL to query OntoStar, the results are shown in Fig 4.

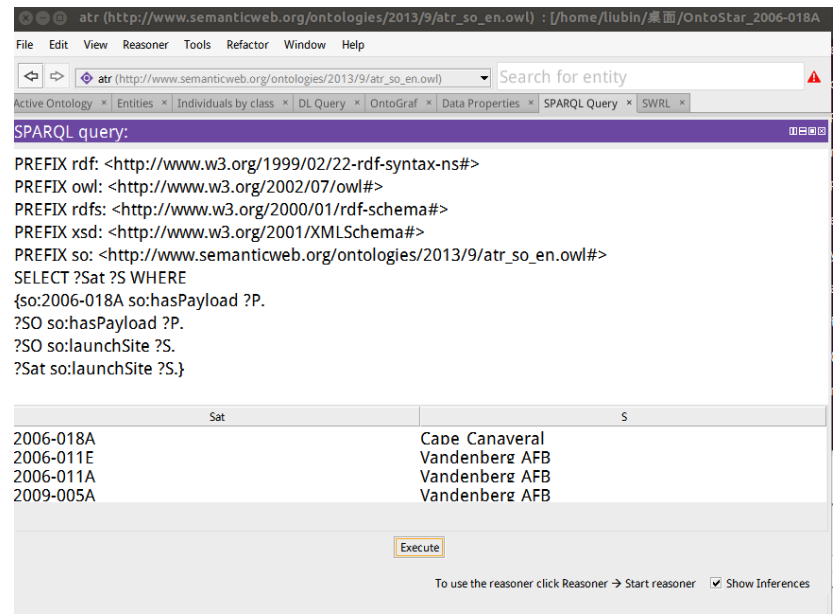

Fig. 4. Query individuals related to 2006-018A.

As can be seen from Fig 4, the launch site of 2006-018A is Cape Canaveral. There are 3 RSOs who owns the same payload as 2006-018A does. They are 2006-011E, 2006-011A, and 2009-005A. The launch site is Vandenberg Air Force Base.

It can be seen from the above examples that the RSO data from multiple sources are linked together by OntoStar, making it convenient to access and analyze RSO data. Using semantic association rules mining ${ }^{(17)}$, interesting relations between the multi-source data can be uncovered by rules obtained.

\subsection{Conducting 2-phase RSO Recognition}

After multi-source data of an RSO are represented in OntoStar, the RSO then can be classified by reasoning on OntoStar. However, too many data may also lead to inconsistent conclusions, making it impossible to give a right result ${ }^{(2)}$. Using multi-source data in classifying RSO also probably encounters this case, because multi-source data contain many correlated features, and the soft data may be inaccurate, deriving the RSO to exclusive classes.

In order to solve the problem of inability to derive RSO types caused by missing features due to incomplete observations, or inconsistent conclusions due to too many multi-source data, we propose 2-phase RSO recognition. The main idea of 2-phase RSO recognition is that, hard data is preferentially used in RSO classification, since soft data is more inaccurate. When limited and uncertain observations result in missing features, that is, hard data cannot be measured and RSO types cannot be deduced thoroughly, then soft data is useful for RSO classification. In summary, the 2-phase RSO recognition is shown below. 
2-phase_RSO_Recognition(OntoStar, hard_data, soft_data)

/*classify RSOs based on reasoning on OntoStar in 2 phases*/

Input: OntoStar, hard_data, soft_data //input soft\&hard data of the RSO to be recognized and the ontology OntoStar for RSO recognition

Output: clsSet //classes which the RSO belongs to

/*1st phase: represent hard data by OntoStar and classifying RSO based on reasoning on OntoStar*/

OntoStar $\leftarrow$ represent(OntoStar, hard_data) $/ /$ represent hard data

If isConsistent(OntoStar) $=$ false

Return null//do not classify the RSO if inconsistency is deduced

Else

axiomSet $\leftarrow$ reason(OntoStar)//infer new axioms from OntoStar clsSet $\leftarrow$ obtainCls(axiomSet)//obtain results from the inferred axioms

If containsTheMostSpecificType(clsSet, OntoStar) = true

Return clsSet//classify RSO thoroughly using hard data

Else skip to the second phase

/*2nd phase: use hard \& soft data by OntoStar for classifying RSO*/ OntoStar $\leftarrow \operatorname{add}($ OntoStar, axiomSet) //add the inferred statements to OntoStar in order to deploy these axioms directly for reasoning in the next OntoStar $\leftarrow$ represent(OntoStar, soft_data) //represent soft data

If isConsistent(OntoStar) $=$ false //if inconsistency is deduced

Return clsSet //classify the RSO only using hard data

Else

axiomSet $\leftarrow$ reason(OntoStar)//infer new axioms from OntoStar

clsSet $\leftarrow$ clsSet $\cup$ obtainCls $($ axiomSet $) / /$ obtain results

Return clsSet//return classes which the RSO belongs to

In the above algorithm, OntoStar-based RSO classification is divided into 2 phases. The first phase uses OntoStar to represent RSO's hard data and to reason types of the RSO. If the RSO is completely classified, the recognition result is returned; otherwise it enters the second phase. The second phase adds the inferred axioms obtained in the first phase to OntoStar, to be used as the known conditions for the forthcoming reasoning. After RSO's soft data are represented by OntoStar, reasoning is performed similar to the first phase to return results.

\section{Experiments of RSO Recognition}

This section implements the RSO recognition system Clairvoyant based on OntoStar first. Then Clairvoyant is used to conduct 2 experiments of RSO recognition.

\subsection{Implementing Clairvoyant}

In order to recognize RSOs based on OntoStar in batch and automatically, an OntoStar-based system named Clairvoyant for RSO recognition was developed. This system represents RSO multi-source data using OntoStar and then reason on OntoStar to classify RSO. The system contains a data interface, which imports the RSO multi-source data to OntoStar representation. By integrating Pellet ${ }^{(20)}$, an inference engine, the system can reason on OntoStar to classify RSOs and convert the inference process into a tree structure.

Clairvoyant is developed using Java. The interface uses Java Database Connectivity (JDBC) to query MySQL database and manipulates OntoStar using OWL-API. OWL-API is an OWL ontology programming development interface that provides interfaces to Pellet, ontology operations, and ontology queries. After accessing the RSO data in MySQL database, OWL-API creates an RSO instance in OntoStar. The query results are parsed into assertions which are added to OntoStar by OWL-API. On this basis, Clairvoyant calls Pellet to reason on OntoStar to obtain the most specific type which the RSO belongs to. A snapshot of Clairvoyant is shown in Fig 5.

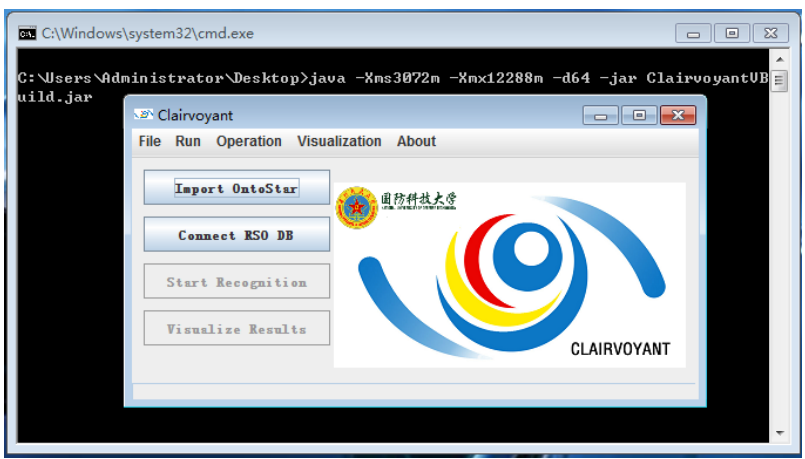

Fig. 5. A snapshot of Clairvoyant.

As can be seen from Fig 5, Clairvoyant provides settings for using OntoStar of different versions, connecting RSO database and importing from data records, recognizing RSOs and visualizing results. To achieve the second phase of the 2-phase RSO recognition, Clairvoyant also provides the option to automatically collect RSO soft data from multiple data sources, as shown in Fig 6.

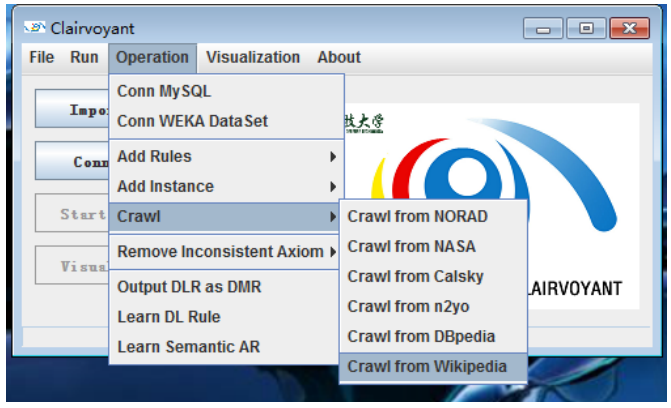

Fig. 6. Collecting soft data in Clairvoyant.

In Fig 6, the data collected from data sources such as 
NORAD, NASA, Calsky, and n2yo are mainly historical data. Data collected from DBpedia and Wikipedia are mainly about RSO-related entities, thus are soft data.

Clairvoyant can assist decision makers of space situational awareness in classifying RSO and understanding the classification process. It provides data input of two alternatives, namely, importing RSO hard data from database and collecting multi-source data to be represented by OntoStar. Based on this, RSO classification based OntoStar reasoning can be implemented in a more flexible manner which is the 2-phase RSO recognition. The setting for using different versions of OntoStar makes it easy to upgrade Clairvoyant without modifying other modules of the system. Thus, the system's degree of modularity is high.

\subsection{Recognizing RSOs Using Clairvoyant}

In RSO catalogs, there are still a large number of unclassified RSOs. Clairvoyant can be used to classify them. E.g., in NORAD Catalog, RSOs are only labeled as satellites, debris, and rockets, and there are a large number of unlabeled RSOs. Among the data published by Calsky, there are some newly cataloged RSOs whose type is not yet known. Using Clairvoyant to collect multi-source data and to reason on OntoStar, some RSOs can be classified.

\section{Classify an unlabeled RSO in Catalogs}

Taking the RSO whose NORAD ID is 39026 (COSPAR ID is 2012-072A, abbreviated as 39026) as the example, it is unlabeled in multiple RSO catalogs. The data sources for the RSO are shown in Table 2.

In Table 2, the bold data can be regarded as soft data. When Clairvoyant cannot classify 39026 thoroughly, these data will be represented by OntoStar in classification. The plain data are treated as hard data and used preferentially.

Table 2. Multi-source data for classifying 39026.

\begin{tabular}{|c|c|}
\hline scheme & data \\
\hline $\begin{array}{l}\text { (norad_id, cospar_id, name, source, period, inclination, apogee, perigee, } \\
\text { eccentricity, rcs, size, amr, launch date) }\end{array}$ & $\begin{array}{l}\text { (39026, 2012-072A, KMS 3-2, NKOR, 95, 97, 557, 487, 0.01, 0.49, 0.74, 0.01, } \\
\text { 2012-12-12) }\end{array}$ \\
\hline TLE Format & $\begin{array}{llllllll}1 & 39026 \mathrm{U} & 12072 \mathrm{~A} & 12347.09611576 & -.00000066 & 00000-0 & 00000+0 & 0 \\
9996239026 & 097.4047 & 036.0317 & 0067405 & 176.3492 & 277.5861 & 15.08261084 & 19\end{array}$ \\
\hline $\begin{array}{l}\text { (cospar_id, norad_id, multiple name flag, payload flag, operational status } \\
\text { code, satellite name(s), source or ownership, launch date, launch site, } \\
\text { decay date, orbital period, inclination, apogee, perigee, rcs) }\end{array}$ & $\begin{array}{l}\text { (2012-072A, 39026, M, * , } \text { KMS 3-2, NKOR, 2012-12-12, YUN, -, 95.0, 97.3, } \\
557,484,0.4890)\end{array}$ \\
\hline $\begin{array}{l}\text { (cospar_id, norad_id, users, dry mass, launch date,launch site, launch } \\
\text { vehicle, purpose) }\end{array}$ & $\begin{array}{l}\text { (2012-072A, 39026, Unknown (North Korea), 100, 2012-12-12, Sohae, North } \\
\text { Korea, Unha, Unknown) }\end{array}$ \\
\hline
\end{tabular}

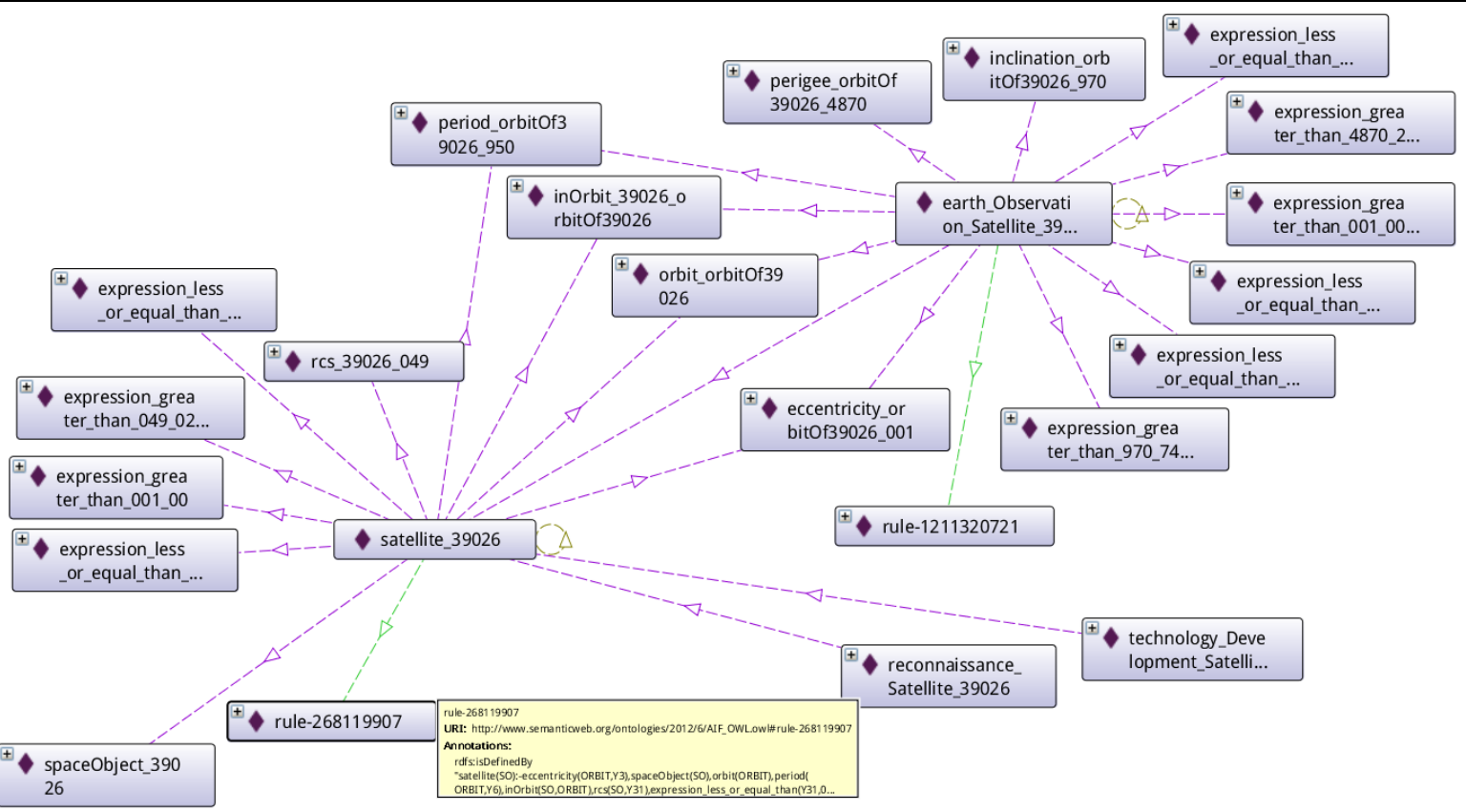

Fig. 7. Inference process of recognizing 2012-072A by Clairvoyant.

Using the hard data in Table 2, 39026 was deduced as an earth observation satellite, technology development satellite, and reconnaissance satellite by Clairvoyant. The reasoning process in classifying it as an earth observation 
satellite is converted into a tree structure shown in Fig 7.

In Fig 7, 39026 is classified step by step. First, using rcs, period, eccentricity, and the rule "rule-268119907", 39026 was deduced to be a satellite by starting from SpaceObject. Then, using period, eccentricity, perigee height, inclination, and the rule "rule-1211320721", 39026 was derived to be an earth observation satellite, with the precondition that 39026 is a satellite. Among the rules, "rule-268119907" is as follows:

spaceObject(SO), orbit $(O), \operatorname{inOrbit}(S O, O), \operatorname{period}(O, V 6)$,

less_or_equal_than(V6,101.02), greater_than(V5,0.28),

less_or_equal_than(V5,0.53), eccentricity $(O R, V 3)$,

$\operatorname{rcs}(S O, V 5)$, greater_than $(V 3,0.0) \rightarrow$ Satellite $(S O)$.

This rule was mined from data which were from 2 sources. It was integrated into OntoStar for recognition.
Using only hard data, 39026 was completely classified by Clairvoyant. Therefore, soft data is not invoked by Clairvoyant in the classification.

\section{Conducting 2-phase RSO Recognition}

The lack of RSO data often occurs due to limited and uncertain observations. Taking the RSO whose NORAD ID is 28500 (COSPAR ID is 2004-050A, abbreviated as 28500) as the example, multiple RSO catalogs do not include the data of the RSO, such as the CEOS payload dataset and the UCS satellite dataset. In the data source including 28500, the type of 28500 is unknown. Data about 28500 are shown in Table 3.

Table 3. Multi-source data for recognizing 28500.

\begin{tabular}{|c|c|}
\hline scheme & data \\
\hline (norad_id, cospar_id, name, source, launch date) & (28500, 2004-050A, DELTA 4 DEMO SPACECRAFT, US, 2004-12-21) \\
\hline TLE & 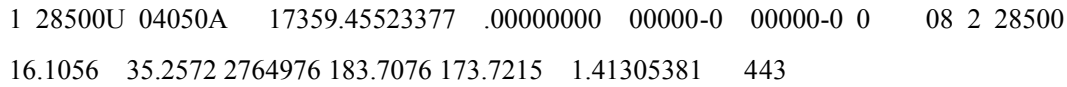 \\
\hline $\begin{array}{l}\text { (norad_id, cosparid, rcs, length, width, height, } \\
\text { radius, period, inclination, perigee, apogee, shape) }\end{array}$ & $(28500,2004-050 \mathrm{AO}, 20,4,2,1019,14,18025,36312$, cylindrical) \\
\hline $\begin{array}{l}\text { (cospar_id, name, dry mass, launch date, launch } \\
\text { site, launch vehicle) }\end{array}$ & (2004-050A, Demosat, 6100, 2004-12-21, Cape Canaveral, Delta IV) \\
\hline
\end{tabular}

First, Clairvoyant uses the hard data (in plain fonts) in Table 3 to deduce the type of 28500. Due to the lack of key features such as size and amr, the type of 28500 cannot be further derived. Then, using soft data (in bold fonts), 28500 is deduced as a communication satellite. The reasoning uses 3 rules. The first rule uses dry mass to deduce the RSO as a satellite, as follows:

SpaceObject(?SO), dryMass(?SO, ?DM), greater_than(?DM, 100.0f) $\rightarrow$ Satellite(?SO)

The second rule guesses user type of RSO. It is as follows:

Satellite(?S), Place('Cape Canaveral'), launchSite(?S, 'Cape Canaveral'), shape(?S,?SH), Cylindrical(?SH), inOrbit(?S, ?OR), GEO (?OR), user(?S, ?M) $\rightarrow$ Military(?M)

The third rule infers satellite type using launch site, launch vehicle, orbit class and user type, as follows:

Satellite(?S), Place('Cape Canaveral'), launchSite(?S, 'Cape Canaveral'), launchVehicle(?S, 'Delta 4'), inOrbit(?S, ?OR), GEO(?OR) , user(?S, ?M), Military(?M)

$\rightarrow$ Communications_Satellite(?S)

Since the second and third rules are derived from soft data, soft data have to be used in triggering the rules. Clairvoyant speculates that 28500 is a communication satellite. The conclusion is only a preliminary estimation. Further confirmation is necessary for decision making.

\section{Conclusions}

This paper proposes to combine the hard data obtained by physical sensors with the soft data obtained from intelligence for RSO classification, targeting at obtaining more accurate classifications under limited and uncertain observation conditions. The RSO ontology for recognition named OntoStar is used to integrate hard data and soft data, and to develop a recognition system named Clairvoyant. Experimental results show feasibility of RSO recognition using Clairvoyant under the conditions of limited and uncertain observations.

Using OntoStar to link various "data silos", a "semantic web" is formed to describe RSO with the RSO as the center. Because reasoning based on OntoStar uses multi-source data and knowledge base, a leap from the "data silos" to the "intelligent web" for RSO recognition is completed.

The 2-phase RSO recognition is proposed to provide preliminary and feasible solutions for recognizing RSOs when lacking RSO data due to incomplete observations, or 
encountering inconsistent conclusions due to too many correlated features and inaccurate soft data.

Clairvoyant, the RSO recognition system based on OntoStar, is implemented. Clairvoyant can be used for 2-phase RSO recognition. Using Clairvoyant to perform RSO classification experiments, it is shown that the 2-phase RSO recognition can integrate hard data and soft data, and obtain more accurate RSO recognition results under limited and uncertain observation conditions. In the future, we will study how to make better use of multi-source data and improve the effectiveness of RSO classification using OntoStar with hard data and soft data.

\section{Acknowledgment}

The presented work is framed within the National Natural Science Foundation of China (No. 71371184).

\section{References}

(1) Furfaro R, Linares R, Gaylor D, Jah M, Walls R: "Resident Space Object Characterization and Behavior Understanding via Machine Learning and Ontology-based Bayesian Networks", Advanced Maui Optical and Space Surveillance Technologies Conference, 2016

(2) Liu B, Yao L, Han D: "Harnessing ontology and machine learning for RSO classification", SpringerPlus, $5,1,1655,2016$

(3) Ruttenberg BE, Wilkins MP, Pfeffer A: "Reasoning on resident space object hierarchies using probabilistic programming", 18th International Conference on Information Fusion (Fusion), 15-21, 2015

(4) Wang JC, Zhou SW, Shi L, Hu YH, Wang Y: "Image quality analysis and improvement of Ladar reflective tomography for space object recognition", Optics Communications, 359, 177-83, 2016

(5) Linares R, Crassidis JL, Jah MK, editors: "Space object classification and characterization via Multiple Model Adaptive Estimation", 17th International Conference on Information Fusion (FUSION), 2014

(6) DeMars KJ, McCabe JS, Darling JE, editors: "Efficient multi-sensor data fusion for space surveillance", American Control Conference (ACC), 2015

(7) Jia B, Pham KD, Blasch E, Shen D, Wang Z, Chen G: "Cooperative space object tracking using space-based optical sensors via consensus-based filters", IEEE Transactions on Aerospace and Electronic Systems, 52,
4, 1908-36, 2016

(8) Zhang L, Wu X, Zhu H, AbouRizk SM: "Perceiving safety risk of buildings adjacent to tunneling excavation: An information fusion approach", Automation in Construction, 73, 88-101, 2017

(9) Khaleghi B, Khamis A, Karray FO, Razavi SN: "Multisensor data fusion: A review of the state-of-the-art", Information Fusion, 14, 1, 28-44, 2013

(10)Hall DL, Mcneese M, Llinas J, Mullen T, editors: "A framework for dynamic hard/soft fusion", International Conference on Information Fusion, 2008.

(11)Blasch EP, Dorion E, Valin P, Bosse E, Roy J, editors: "Ontology alignment in geographical hard-soft information fusion systems", Information Fusion, 2010.

(12)Corry E, O’Donnell J, Curry E, Coakley D, Pauwels P, Keane M: "Using semantic web technologies to access soft AEC data", Advanced Engineering Informatics, 28, 4, 370-80, 2014

(13)Sweet N, Ahmed N, editors: "Structured synthesis and compression of semantic human sensor models for Bayesian estimation", American Control Conference, 2016

(14)Gross GA, Date K, Schlegel DR, Corso JJ, Llinas J, Nagi R, et al., editors: "Systemic test and evaluation of a hard+soft information fusion framework: Challenges and current approaches", 17th International Conference on Information Fusion (FUSION), 2014

(15)Cox AP, Nebelecky CK, Rudnicki R, Tagliaferri WA, Crassidis JL, Smith B: "The Space Object Ontology", 19th International Conference on Information Fusion (FUSION), 2016

(16)Rovetto RJ: "An ontology for satellite databases", Earth Science Informatics, 1-11, 2017

(17)Fürnkranz J, Kliegr T, editors: "A Brief Overview of Rule Learning", RuleML2015, 2015

(18)Fürnkranz J: "Classification Rule. In: Sammut C, Webb GI, editors. Encyclopedia of Machine Learning and Data Mining", Boston, MA: Springer US, 209, 2016

(19)Sirin E, and Bijan Parsia: "SPARQL-DL: SPARQL Query for OWL-DL", OWLED, 2007

(20)Sirin E PB, Grau B C, et al: "Pellet: A practical owl-dl reasoner", Web Semantics: science, services and agents on the World Wide Web, 5, 2, 51-73, 2007 